\title{
Simulation to Improve Trainee Skill and Comfort with Forceps-Assisted Vaginal Deliveries
}

\author{
Kelsey Rose, $\mathrm{MD}^{1} \quad$ Kirsten Jensen, $\mathrm{MD}^{1} \quad$ Rong Guo, MS ${ }^{2} \quad$ Yalda Afshar, MD, PhD ${ }^{1}$ \\ 1 Department of Obstetrics and Gynecology, David Geffen School of
Medicine, University of California, Los Angeles, California
2Department of Medicine Statistics Core, David Geffen School of
Medicine, University of California, Los Angeles, California \\ Address for correspondence Yalda Afshar, MD, PhD, Division of \\ Maternal Fetal Medicine, Department of Obstetrics and Gynecology, \\ David Geffen School of Medicine, University of California, Los Angeles, \\ 10833 Le Conte Avenue, Room 27-139 CHS, Los Angeles, \\ CA 90095-1740 (e-mail: yafshar@mednet.ucla.edu).
}

Am J Perinatol Rep 2019;9:e6-e9.

\begin{abstract}
Keywords

- forceps-assisted vaginal delivery

- obstetrics

- simulation

- medical education

- operative delivery

Objective Simulation training is a powerful learning tool for low frequency events. Forceps-assisted vaginal deliveries (FAVD) are an important tool in reducing cesarean deliveries. The aim of this study is to create a high-fidelity simulation-based curriculum for residency education and investigate pre- and posttest skill and confidence.

Methods A prospective cohort study was conducted involving obstetrics and gynecology residents over 2 academic years. Residents participated in one to three FAVD simulation trainings. All sessions involved video, didactic, and hands-on practice. Pre- and postsurvey and skills assessment were conducted to assess confidence, ability to consent, and perform a FAVD. Wilcoxon's signed-rank tests and Kruskal-Wallis tests were used.

Results Thirty residents (73\%) completed at least one forceps simulation training session. Participants demonstrated significant improvement in confidence $(p<0.005)$ following training. Before the intervention, there was a disparity in confidence by postgraduate level $(p<0.005)$; however, this difference was not seen postsimulation $(p=0.24)$. Residents demonstrated significant improvement in their FAVD skills $(p<0.05)$, as well as their ability to consent $(p<0.01)$.

Conclusion Simulation training improves residents' perceived confidence in FAVD. Simulation helped to better equalize confidence across classes. FAVD simulations improves resident confidence, skill, and more broadly broadened the armamentarium to decrease the cesarean delivery rate.
\end{abstract}

Simulation training, as a learning tool, has been used frequently and extensively over the past century. ${ }^{1}$ First used in aviation for flight simulations in the 1910s, simulators designed to mimic patients were developed in the 1960s. Today, simulation is an integral tool for training in the medical field. ${ }^{2}$ Anesthesia specifically has historically used simulation exercises most comprehensively. ${ }^{3}$ McGaghie et al conducted a meta-analysis of studies over 20 years, demonstrating that simulation is superior for skill acquisition than traditional didactic learning. As they point out, the power and value of simulation is no longer in question. ${ }^{4} \mathrm{~A}$ body of literature has

Dr. Yalda Afshar's ORCID is 0000-0003-3807-7022 shown that simulation is a more effective tool to lecture based learning in regards to improving competency and knowledge among residents and medical students. ${ }^{5}$ In the field of obstetrics, simulation has been shown to help in improving the management of low frequency but high intensity events. ${ }^{6,7}$ Jude et al randomized students to simulation training versus traditional lecture instruction. Of the students who participated in simulation training, $88 \%$ felt ready to do vaginal deliveries independently or with minimal supervision, compared with $12.5 \%$ of those who only received lecture training. ${ }^{5}$ In 2016, Easter et al evaluated the use of simulation training to improve resident knowledge and comfort regarding twin vaginal births. In their study, obstetrics and gynecology

Copyright @ 2019 by Thieme Medical Publishers, Inc., 333 Seventh Avenue, New York, NY 10001, USA. Tel: +1(212) 584-4662.

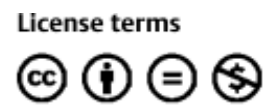

November 12, 2018 accepted after revision November 29, 2018
DOI https://doi.org/ 10.1055/s-0039-1677736 ISSN 2157-6998. 
residents complete counseling module, simulation of twin vaginal delivery, and a breech extraction skills station. Participants in simulation demonstrated significant improvement in knowledge and comfort. ${ }^{8}$ Simulation training interventions in obstetrics trainees with forceps-assisted vaginal deliveries (FAVDs) has not been well published, in an era of low FAVD rates.

FAVDs have declined in prevalence over recent years. ${ }^{9}$ In $2015,3.1 \%$ of all deliveries were operative vaginal deliveries, with forceps deliveries accounting for only $0.6 \%$ of vaginal births. ${ }^{9}$ Operative vaginal deliveries have been highlighted as a key tool to decreasing the primary cesarean delivery rate. $^{10,11}$ There is a significant need to prevent forceps deliveries from becoming a lost art, ${ }^{12,13}$ and simulation training can be an effective way to begin teaching residents once again how to safely and effectively perform FAVDs. The aim of this study is to create a simulation-based curriculum and analyze resident knowledge, confidence, and skill on FAVDs before and after simulation training. Our hypothesis is that our intervention will improve both skill and confidence of obstetrics and gynecology residents.

\section{Materials and Methods}

This was an Institutional Review Board approved (UCLA [University of California, LA] IRB 16-001820) prospective cohort study. All UCLA Obstetrics and Gynecology residents were eligible to participate in three 90-minute simulation training sessions during the 2016 to 2017 and 2017 to 2018 academic years. Participation was voluntary and refusal to participate did not exclude residents from participating in forceps training. Residents completed an anonymous 26 -item pre- and postsimulation confidence survey (see - Supplementary Material S1 and -Supplementary Material S2). Each participant completed a pre- and postsimulation skills assessment graded with a standardized a priori designed checklist. Skills assessments for both consenting of an operative delivery and the forceps deliveries were evaluated by faculty versed and comfortable in FAVD.

Each simulation session started with and ended with a pre- and postsimulation survey assessing residents about their confidence in forceps deliveries. The curriculum at two of the three simulation sessions was the same, while the other had an additional focus of how to consent patients.

For each simulation session, residents were presented with a clinical case that necessitated expedited delivery. In two of the sessions the residents were then required to complete a FAVD using a "Noelle S550 Maternal Care Patient Simulator," which allowed for complete simulation of the operative vaginal delivery experience. The case presented was a low-risk nulliparous woman without a maternal or fetal contraindication to an operative delivery. The resident was told she had an appropriate labor curve, completed dilated, ruptured, with a fetus at +2 station, and an estimated fetal weight of 3,400 grams. Residents' skills at an FAVD were assessed with a premade operative checklist. In the third session, the cohort was required to consent a patient, represented using the Noelle Simulator, for a FAVD. The resident was assessed on their ability to discuss the risks, benefits, and alternatives of a FAVD. Residents included in the study participated in anywhere from one to three of the sessions.

Residents then watched a 5-minute video on forceps and forceps deliveries, or consenting, and then received hands-on training with faculty. After they felt they had sufficient practice in the allotted training time (maximum 45 minutes), they completed a postsimulation assessment of their ability to complete a forceps delivery or consent. Only residents who completed both pre- and postsimulation surveys and skills assessments were included in the study.

Our primary outcome was improvement in resident confidence and skill before and after simulation training. Secondary outcomes included the change in percentage of FAVDs performed at our institution before and after initiation of the forceps simulation-based curriculum.

Standard descriptive statistics were reported (median with the first and third quartile). Wilcoxon's signed ranktest was used for the paired comparison between pre and post confidence and percent correct skill assessment scores. Kruskal-Wallis test was used for the comparison of the improvement score by postgraduate year (PGY). All analyses were performed using SAS 9.4 (SAS Institute Inc., Cary, NC).

\section{Results}

Over the 2 academic years, there was a total of 41 residents eligible to participate. There were 30 residents that participated (73\% of all residents) in at least one session. Residents participated in one to three of the simulation sessions. On average residents had completed none or one FAVD before the simulation.

Residents were evaluated on their confidence performing FAVDs. There was significant improvement in confidence with FAVD pre- versus postsimulation (-Table 1), including those looking at the didactical steps of FAVD and consenting the patient on the procedure. There was no difference in the first session regarding residents' confidence in supervising other residents in FAVD. However, in session two there was a statistically significant improvement, demonstrating that the repetition was a factor in developing the ability to supervise or teach.

Resident's skills were evaluated to pre-and postsimulation with a standardized a priori designed checklist provided to the attending scoring, and there was notably a significant improvement in both skills in FAVD and ability to consent for all residents in all three sessions (-Fig. 1, - Table 2). - Fig. 2 depicts the change by PGY for sessions one and three, as these sessions mirrored each other. There is an improvement by PGY as it related to consenting patients for FAVD (-Fig. 3 ). PGYs 1, 2, and 3 showed a larger degree of change in skill and ability to consent with simulation than PGY 4 (-Table 3 ).

\section{Discussion}

Our simulation successfully improved resident confidence and skill in FAVD and consenting patients for FAVD. Simulation for resident learners is a useful tool for teaching FAVD given the interventions positive impact on both skill and 
Table 1 Change in confidence pre-and postsimulation

\begin{tabular}{|l|l|l|l|l|}
\hline Survey measure & \multicolumn{2}{|l|}{ Session $\mathbf{1}(\boldsymbol{n}=\mathbf{1 4})$} & Session 2 $(\boldsymbol{n}=\mathbf{1 5})$ \\
\hline & $\begin{array}{l}\text { Median improvement } \\
\text { score }\end{array}$ & $\boldsymbol{p}$-Value & $\begin{array}{l}\text { Median improvement } \\
\text { score }\end{array}$ & $p$-Value \\
\hline Identify maternal risks & 1 & 0.0039 & 0 & 0.0469 \\
\hline Identify fetal risks & 1 & 0.0039 & 0 & 0.0156 \\
\hline Identify types of forceps & 1 & 0.0039 & 1 & 0.0137 \\
\hline Identify candidates for FAVDs & 1 & 0.0010 & 1 & 0.0010 \\
\hline Ability to position 1stforcep & 2 & 0.0005 & 1 & 0.0005 \\
\hline Ability to position 2ndforcep & 2 & 0.0005 & 1 & 0.0005 \\
\hline Ability to provide proper traction & 1.5 & 0.0005 & 1 & 0.0215 \\
\hline Ability to know when to remove the forceps & 2 & 0.0005 & 1 & 0.0234 \\
\hline Ability to disengage forceps & 1.5 & 0.0005 & 1 & 0.0010 \\
\hline Ability to discuss risks with family & 1 & 0.0020 & 1 & 0.0078 \\
\hline Ability to supervise other residents in FAVDs & 0 & 0.0625 & 0 & 0.0156 \\
\hline Ability to consent patients for FAVD & - & - & 1 & 0.0020 \\
\hline
\end{tabular}

Abbreviation: FAVD, forceps-assisted vaginal delivery.

amprovement score (range: $0-2$ ) is calculated as the difference of the post session score and the pre session score.

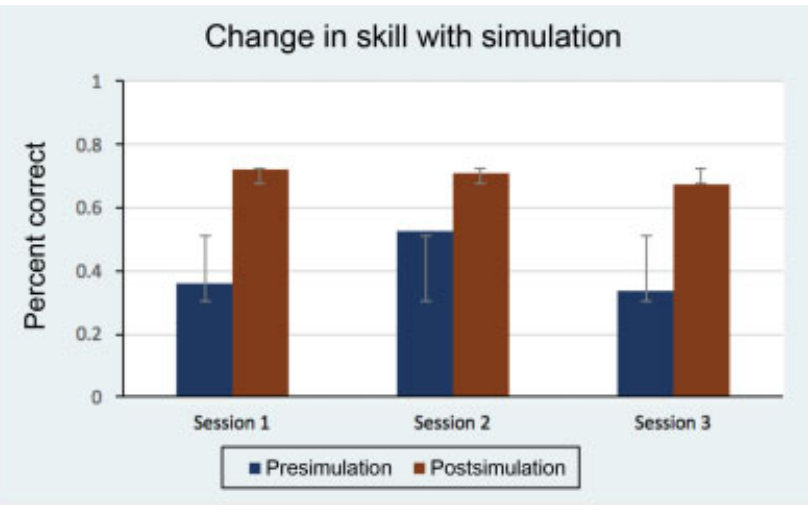

Fig. 1 Change in skill before and after simulation training for all residents.

Table 2 Significant in difference in skills correct for all residents before and after each simulation session

\begin{tabular}{|l|l|l|}
\hline $\begin{array}{l}\text { Difference in skills } \\
\text { correct from pre- to } \\
\text { postsimulation }\end{array}$ & $\begin{array}{l}\text { Median } \\
\text { improvement }_{\text {Score }^{\mathrm{a}}}\end{array}$ & $\begin{array}{l}\text { Difference } \\
\text { between all } \\
\text { residents } \\
(\boldsymbol{p} \text {-value) }\end{array}$ \\
\hline Session 1 & 0.32 & 0.0005 \\
\hline Session 2 & 0.18 & 0.0015 \\
\hline Session 3 & 0.43 & 0.0020 \\
\hline
\end{tabular}

amprovement score is calculated as the difference of the postsession score and the presession score.

confidence. While the simulation does require a Noelle simulation doll, or a similar tool, it is otherwise easy to reproduce and only requires physicians skilled in the art of FAVD to act as teachers and evaluators. After the simulation, residents expressed more confidence in performing FAVDs and were able to perform more of the skills of a FAVD

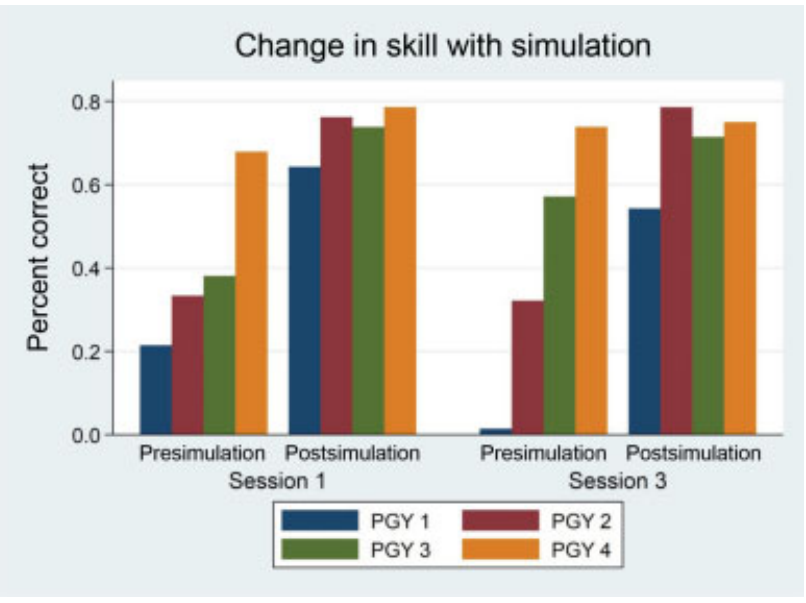

Fig. 2 Change in skill by PGY class in sessions 1 and 3. PGY, postgraduate year.

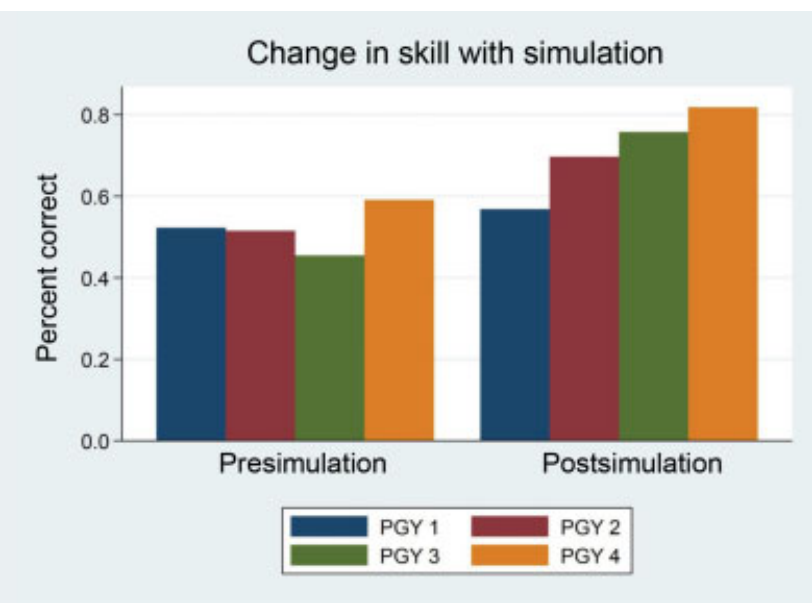

Fig. 3 Change in skills with simulation for session 2 . 
Table 3 Median change in percent of skills performed correctly (sessions 1 and 3 ) and percent of items accurately addressed while consenting pre- and postsimulation by PGY

\begin{tabular}{|l|l|l|l|l|l|}
\hline & $\begin{array}{l}\text { PGY1 } \\
(\%)\end{array}$ & $\begin{array}{l}\text { PGY2 } \\
(\%)\end{array}$ & $\begin{array}{l}\text { PGY3 } \\
(\%)\end{array}$ & $\begin{array}{l}\text { PGY4 } \\
(\%)\end{array}$ & p-Value \\
\hline Session 1 & 39 & 36 & 36 & 11 & 0.24 \\
\hline Session 2 & 5 & 18 & 18 & 27 & 0.14 \\
\hline Session 3 & 57 & 57 & 14 & 11 & 0.11 \\
\hline
\end{tabular}

Abbreviation: PGY, postgraduate year.

Note: The p-values are for the comparison among the PGY groups for each session.

correctly. Additionally, simulation training narrowed the gap between classes in perceived confidence, thus helping to equalize classes. Importantly, the largest difference in improvement in skill was seen in the earlier years of residency, implying that simulation training needs to be done early to have the greatest impact. However, given that most residents perform so few FAVD during training, it could be thought that we should wait, and focusing on training laborists and maternal fetal medicine physicians in FAVD, rather than residents.

Simulation training is an important complement to traditional teaching methods. It allows for practice and preparation, especially with events that occur in small volumes. While nothing, including simulation, is a perfect substitute for real life experiences, simulation allows us to mimic real life, making us feel prepared for the challenges we face in many acute situations. Simulation allows residents to have practice in the wide variety of procedures performed in the field of obstetrics and gynecology. In one example, as FAVDs decreases, simulation training becomes necessary to maintain resident exposure and comfort. Studies have shown that simulation training, in general and specifically with regards to FAVD, improves patient safety. Not only does simulation improve resident confidence and skill but this translates to better patient outcomes, though is still associated with a $30 \%$ risk of severe perineal trauma. ${ }^{14}$

The primary limitations of our study include the small sample size and single institution sample. Additionally, this study demonstrates a short term and immediate change in skill and comfort but does not establish if this change persists over time. Further studies would need to be done to compare resident confidence and immediately skill after a simulation session and throughout training. Our study also demonstrates selection bias, given that residents who wanted to learn FAVD were more likely to participate. Given that we were limited to perform these simulation sessions during scheduled resident teaching time, we did not want to prevent any residents from having the opportunity to learn and thus did not have a control group that would not receive the FAVD simulation training. Finally, while this study did improve perceived confidence and skill at FAVD simulation, we did not show or evaluate improvement in skill level in an actual FAVD or clinical benefit.

\section{Conclusion}

In an environment where FAVD have decreased in prevalence and thus resident exposure to such deliveries has decreased, the opportunity for simulation training becomes vital. Incorporating simulation session with FAVD will help to improve resident confidence and skill. Further studies should assess the improvement in resident knowledge on FAVD with simulation.

\section{Note}

There are no financial disclosures.

Conflict of Interest

None.

\section{References}

1 Satin AJ. Simulation in obstetrics. Obstet Gynecol 2018;132(01): 199-209

2 Page RL. Brief history of flight simulation. Available from: http:// citeseerx.ist.psu.edu/viewdoc/download?doi=10.1.1.132.5428\& rep $=$ rep1\&type $=$ pdf Accessed December 27, 2018

3 Gardner R, Raemer DB. Simulation in obstetrics and gynecology. Obstet Gynecol Clin North Am 2008;35(01):97-127, ix

4 McGaghie WC, Issenberg SB, Cohen ER, Barsuk JH, Wayne DB. Does simulation-base medical education with deliberate practice yield better results than traditional clinical education? A metaanalytic comparative review of the evidence. Acad Med 2011;86 (06):706-711

5 Jude DC, Gilbert GG, Magrane D. Simulation training in the obstetrics and gynecology clerkship. Am J Obstet Gynecol 2006; 195(05):1489-1492

6 Nielsen PE, Goldman MB, Mann S, et al. Effects of teamwork training on adverse outcomes and process of care in labor and delivery: a randomized controlled trial. Obstet Gynecol 2007;109(01):48-55

7 Siassakos D, Crofts JF, Winter C, Weiner CP, Draycott TJ. The active components of effective training in obstetric emergencies. BJOG 2009;116(08):1028-1032

8 Easter SR, Gardner R, Barrett J, Robinson JN, Carusi D. Simulation to improve training knowledge and comfort about twin vaginal birth. Obstet Gynecol 2016;128(Suppl 1):34S-39S

9 Martin JA, Hamilton BE, Osterman MJ, Driscoll AK, Mathews TJ. Births: final data for 2015. Natl Vital Stat Rep 2017;66(01):1

10 American College of Obstetricians and Gynecologists; Society of Maternal-Fetal Medicine, Caughey; Caughey AB, Cahill AG, Guise J-M, Rouse DJ. Safe prevention of the primary cesarean delivery. Am J Obstet Gynecol 2014;123:693-711

11 Shaffer BL, Caughey AB. Forceps delivery: potential benefits and a call for continued training. J Perinatol 2007;27(06):327-328

12 Gupta N, Dragovic K, Trester R, Blankstein J. The changing scenario of obstetric and gynecology residency training. J Grad Med Educ 2015;7(03):401-406

13 Dildy GA, Belfort MA, Clark SL. Obstetric forceps: a species on the brink of extinction. Obstet Gynecol 2016;128(03):436-439

14 Gossett DR, Gilchrist-Scott D, Wayne DB, Gerber SE. Simulation training for forceps-assisted vaginal delivery and rates of maternal perineal trauma. Obstet Gynecol 2016;128(03):429-435 